\title{
Comparison of the Hardness of Novel Experimental Vinyl Poly Siloxane (VPS) Impression Materials with Commercially Available Ones
}

\author{
Shahab Ud Din $\mathbb{D}^{1,}$, ${ }^{1,2}$ Farooq Ahmad Chaudhary $\mathbb{D}^{1}{ }^{1}$ Bilal Ahmed, \\ Mohammad Khursheed Alam $\left(D,{ }^{3}\right.$ Sandra Parker, ${ }^{2}$ Mangala Patel, \\ and Muhammad Qasim Javed $\mathbb{C}^{4}$ \\ ${ }^{1}$ School of Dentistry (SOD), Federal Medical Teaching Institution (FMTI)/PIMS, Shaheed Zulfiqar Ali Bhutto Medical \\ University (SZABMU), Islamabad, Pakistan \\ ${ }^{2}$ Centre for Oral Bioengineering (Dental Physical Sciences Unit), Bart's and the London School of Medicine and Dentistry, \\ Queen Mary University of London, UK \\ ${ }^{3}$ Preventive Dentistry Department, College of Dentistry, Jouf University, 72345 Sakaka, Saudi Arabia \\ ${ }^{4}$ Department of Conservative Dental Sciences, College of Dentistry, Qassim University, Al-Qassim, Saudi Arabia
}

Correspondence should be addressed to Shahab Ud Din; drshahab728@hotmail.com

and Farooq Ahmad Chaudhary; chaudhary4@hotmail.com

Received 16 July 2021; Revised 14 October 2021; Accepted 26 January 2022; Published 9 February 2022

Academic Editor: Victor Feitosa

Copyright (c) 2022 Shahab Ud Din et al. This is an open access article distributed under the Creative Commons Attribution License, which permits unrestricted use, distribution, and reproduction in any medium, provided the original work is properly cited.

\begin{abstract}
Purpose. To determine the hardness and Young's moduli of both commercial and experimental vinyl poly siloxane (VPS). Methods. The purpose of this study was to develop a medium-bodied experimental (Exp-I, II, III, IV, and V) VPS impression materials and to analyse their effects on hardness and Young's modulus and compare them with three commercial VPS materials (Aquasil, Elite, and Extrude) using Shore A hardness tester. Measurements were recorded after 1, 24, 72, and 168 hours of mixing. The results were analysed with one-way ANOVA and post hoc Tukey's test using the SPSS PASW statistical 22 software. Results. Commercial and experimental vinyl polysiloxane exhibited higher Shore A hardness values with time (i.e., 1 hour after mixing, 24 hours after mixing, 72 hours after mixing, and 1 week after mixing). All Comml and Exp VPS demonstrated a significant increase (ANOVA, $p<0.05$ ) in hardness at increasing time points. Generally, all commercial VPS exhibited significantly higher values for Shore A hardness compared to all Exp formulations. For commercial products, Elt $\mathrm{M}$ presented significantly highest values at all-time points followed by Aq M then Extr M. Exp-I was significantly harder than all other Exp VPS at all-time points. Young's modulus values were directly related to Shore A hardness; materials with higher Shore A hardness values had higher Young's moduli. Conclusion. Continued polymerisation of elastomeric impression materials results in increased hardness over time. Hardness, Young's moduli, and rigidity of the set commercial and experimental VPS materials were within the required limits. Shore A hardness and Young's moduli were directly proportional to each other, and commercial and experimental materials had enough rigidity to contain the stone during pouring.
\end{abstract}

\section{Introduction}

The ability of a material to resist surface indentation or penetration is called its hardness [1]. A variety of tests are available to measure hardness. These tests are defined by the geometry and dimensions of their indenters and the amount of load applied. The load per unit surface area of the indentation gives the hardness number [2,3]. Brinell, Knoop, Vicker, Rockwell, and Barcol are used to measure the hardness of rigid materials such as metals, alloys, and rigid 
restorative dental materials. Unfortunately, these methods cannot be used for elastic materials, such as elastomers, where the deformation is elastic rather than permanent [1].

The force necessary to remove the impression from the mouth is directly related to the hardness of the impression material [1]. The hardness also changes with time for some materials (e.g., vinyl polysiloxane: Aquasil). Elastomers are characterised according to their hardness. This is why it is one of the most important and most studied characteristics of these materials. The most commonly used methods are the International Rubber Hardness Degrees (IRHD) and Shore hardness test. A wide range of Shore durometers has been described by ASTM D2240 with Shore A hardness being the most appropriate for the measurement of hardness of elastomeric impression materials. The Shore A hardness scale increases from low to high viscosity $[4,5]$.

1.1. Relation between Hardness and Young's Modulus. The ability of a material to resist deformation under stress due to its stiffness is referred to as its Young's or elastic modulus [6]. Young's modulus of an elastomeric impression material can be calculated from its hardness value. Meththananda et al. evaluated the relationship between hardness and Young's moduli of some elastomeric impression materials where they measured the Shore A hardness of VPS and one polyether impression material [2]. Young's moduli were calculated from the hardness values using the following equations: $H=100 \operatorname{erf}\left(k E^{1 / 2}\right)$, where $H$ is hardness value, $k$ is $3.186 \times 10^{-4} \mathrm{~Pa}^{-1 / 2}, E$ is Young's modulus, and the erf is the error function (to generate a hardness scale), and

$$
E(\mathrm{MPa})=\frac{0.0981(56+7.66 \mathrm{~s})}{0.137505(254-2.54 \mathrm{~s})},
$$

where $s$ is Shore A hardness. The direct method is a measure of stress/strain in tension. The first equation gave more accurate values (closer to the direct method). The two equations were compared with each other and with the Young's moduli calculated by the direct method (Table 1) [2, 7].

The elastic modulus of elastomeric impression materials of similar viscosity increases in the following order: polysulphides, condensation silicone, VPS, and polyether [8].

Six commercially available impression materials, three VPS (Aquasil light, Honigum light, President Plus Jet), two condensation silicone (Rapid Liner, Detaseal light), and one polyether (Impregum F), were evaluated by Papadogiannis et al. for Young's modulus [9]. The materials were stored at room temperature $\left(22^{\circ} \mathrm{C}\right)$ for $30 \mathrm{~min}, 60 \mathrm{~min}, 3$ hours, 24 hours, 48 hours, 1 week, and 2 weeks period time. Their results showed that Young's moduli increased for all materials investigated with increasing storage time. The Young's moduli ranged from 1.81 to $12.99 \mathrm{MPa}$, with the polyether material being the stiffest $(10.63-12.56 \mathrm{MPa})$, followed by VPS and finally condensation silicones.

The objectives of this study were to develop novel experimental VPS impression materials to improve their hardness, other mechanical, and wetting properties. The details about the other mechanical properties such as tear strength, tensile strength, and wetting properties are given in the pub- lished part of this study [10-13]. The newly formulated five experimental VPS impression materials containing an additional cross-linking agent (tetra-functional (dimethylsilyl) orthosilicate (TFDMSOS)) and a novel surfactant, Rhodasurf CET-2 (ethoxylated cetyl-oleyl alcohol), were compared with three commercial VPS impression materials. The hypothesis of the current study was that the additional cross-linking agent (TFDMSOS) will form a further bond of crosslinking leading to improvement in the mechanical properties as it happened in the cases of tear strength [11, 13] and tensile strength [10]. The novel surfactant (Rhodasurf CET-2) would also chemically bond to the polymer matrix as a result of its chemical structure and would further improve the hardness of these materials [11]. It is known that the stiffer impression materials (with high Young's modulus) cause difficulty in removing the impression (and tray) from the mouth $[2,14]$.

It is important to note that strain-in-compression is another sort of hardness test, and the values of strain-incompression of an impression material should be within the normal range of ISO4823 (2007) [15]. Hence, impression materials should not be very stiff yet they should have enough rigidity to contain the stone during pouring.

\section{Materials and Methods}

Hydrophilic commercially available VPS impression materials were used in this study (Table 2) [16]. To standardize the mixing ratios, they were supplied in auto-mixed cartridges of medium-body consistency.

\subsection{Preparation of Experimental VPS Impression Materials.} Tetra-functional dimethylsilyl orthosilicate (TFDMSOS) and Rhodasurf CET-2 which are cross-linking agents were added to improve tear strength. Nonionic surfactant ethoxylated cetyl-oleyl alcohol was added to improve the wetting. Different compositions were prepared, and out of 113 experimental formulations, only five formulations were suitable for use and were labeled (Exp-I, II, III, IV, and V). The formulations and their details are present in the published part of the present research $[16,17]$. For the following formulations, the catalyst paste was the same (Exp-I and II). The control for Exp-II was Exp-I. The Exp-II was used as a control for Exp-III, IV, and V. The formulations for (Exp-III, $\mathrm{IV}$, and V) the catalyst paste were the same.

The hardness of both commercial and experimental was determined by using the Shore A hardness tester (H17A, Congenix Wallace, Kingston, England) in accordance with ASTM:D2240 [17] (Figure 1). Standard rubber test reference blocks supplied by the manufacturers were used to calibrate the equipment before each use.

The Shore A hardness tester is a cylindrical indenter measuring $1.6 \mathrm{~mm}$ in diameter; it narrows to a blunt tip measuring $0.8 \mathrm{~mm}$. It is allowed to stabilise for 10 minutes before the test. To minimise the effects of creep, the indenter is pressed on the specimen for one second of dwell time. Measurements were on a scale of 0 to 100 units, and each measurement was at least $10 \mathrm{~mm}$ apart from each other and $12 \mathrm{~mm}$ from the edge of the specimen [17] (Figure 2). 
TABLE 1: Young's moduli of elastomeric impression materials calculated from Shore A hardness values $[2,7]$.

\begin{tabular}{|c|c|c|c|c|c|}
\hline \multirow[b]{2}{*}{ Materials } & \multirow[b]{2}{*}{ Brands } & \multirow[b]{2}{*}{ Shore A hardness } & \multicolumn{3}{|c|}{ Young's modulus (MPa) } \\
\hline & & & $\begin{array}{l}\text { Calc.* direct } \\
\text { method }\end{array}$ & $\begin{array}{l}\text { Calc. error function } \\
\text { (equation in text) }\end{array}$ & $\begin{array}{c}\text { Calc. gent equation } \\
\text { (equation in text) }\end{array}$ \\
\hline \multirow{5}{*}{ VPS } & Zerosil soft & 54.5 & 3.5 & 3.2 & 2.9 \\
\hline & Zerosil super soft & 53.1 & 3.0 & 3.0 & 2.8 \\
\hline & Zerosil light & 56.7 & 3.5 & 3.4 & 3.2 \\
\hline & Zerosil mono & 62.9 & 4.1 & 4.3 & 4.1 \\
\hline & Extrude & 53.2 & 2.6 & 2.6 & 2.8 \\
\hline Polyether & Impregum PS & 53.7 & 2.9 & 2.6 & 2.8 \\
\hline
\end{tabular}

${ }^{*}$ Calculations.

TABLE 2: Commercially available vinyl polysiloxane impression materials used in the study.

\begin{tabular}{lcc}
\hline Commercial VPS & $\begin{array}{c}\text { Lot/batch } \\
\text { number }\end{array}$ & Manufacturers \\
\hline Aquasil ultra monophase (Aq M) & 090505 & Dentsply, USA \\
Elite HD monophase (Elt M) & 95503 & Zhermack, Italy \\
Extrude (Extr M) & $0-1068$ & Kerr, USA \\
\hline
\end{tabular}

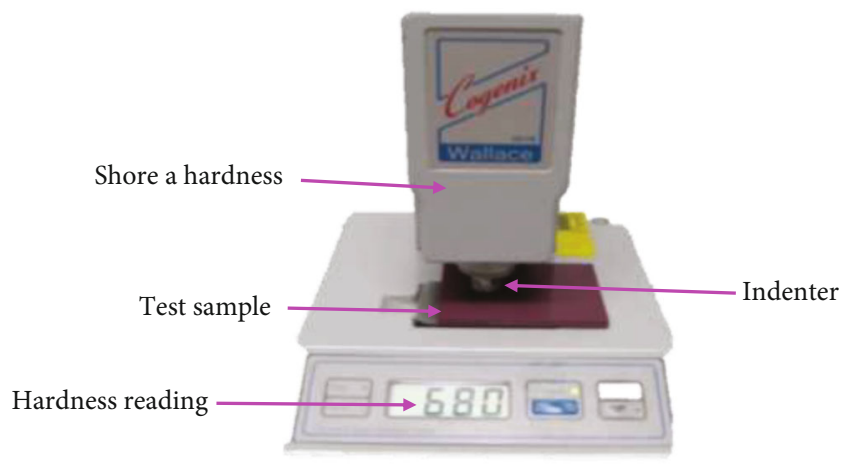

Figure 1: A typical Shore A hardness set up of Aq M in a Shore A.

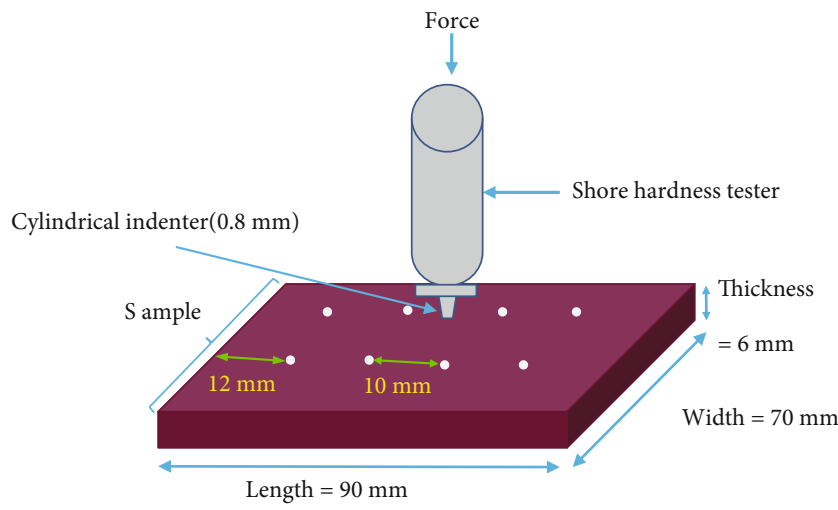

FIgURE 2: Schematic illustration of Shore A hardness indentations on a test sample $(n=12)$.
Shore A hardness number was scored at 100 if no displacement occurred whereas a score of 0 was given to complete penetration. At predetermined time periods, measurements $(n=12$ per sample per material) were taken: 1 hour after mixing, 24 hours after mixing, 72 hours after mixing, and 168 hours (1 week) after mixing $[9,18-20]$.

Shore A hardness values using the following were used to calculate Young's (elastic) moduli at all-time points for all commercial and experimental VPS samples: $H=100$ erf $(k$ $\left.E^{1 / 2}\right)$, where $H$ is Shore A hardness value, $k$ is $3.186 \times 10^{-4}$ $\mathrm{Pa}^{-1 / 2}, E$ is Young's modulus, and the erf is the error function (to generate a hardness scale).

\section{Results}

This study showed a significant increase (ANOVA, $p<0.05)$ in hardness at increasing time points (1 hour after mixing, 24 hours after mixing, 72 hours after mixing, and 168 hours after mixing) for all commercial and experimental VPS and can be appreciated in Figure 3.

All Exp formulations showed significantly lower values of Shore A hardness as compared to commercial VPS. At all-time points studied for commercial products, Elt $\mathrm{M}$ showed the highest values followed by Aq M then Extr M. Exp-I was found to be significantly harder than all other Exp VPS. The hardness of Exp-II decreased as compared to Exp-I (control), by the addition of TFDMSOS and was a further significant $(p<0.001)$ decrease after the addition of the surfactant into Exp-III, IV, and V. The Shore A hardness decreased for Exp-III, IV, and V at all-time points as the concentration of surfactant $(2 \%, 2.5 \%$, and $3 \%)$ increases. There was no significant difference between ExpIV and V at 24 hours and Exp-III, IV, and V at 168 hours after mixing (Figure 3).

It has been found that Shore A hardness and Young's moduli are directly proportional to each other as Young's moduli for all materials were calculated from Shore A hardness values (Figure 4).

\section{Discussion}

The novel experimental vinyl polysiloxane impression materials had comparatively lower hardness and Young's modulus yet these materials were within the normal range of stain-in-compression according to ISO4823 (2007) [15]. 


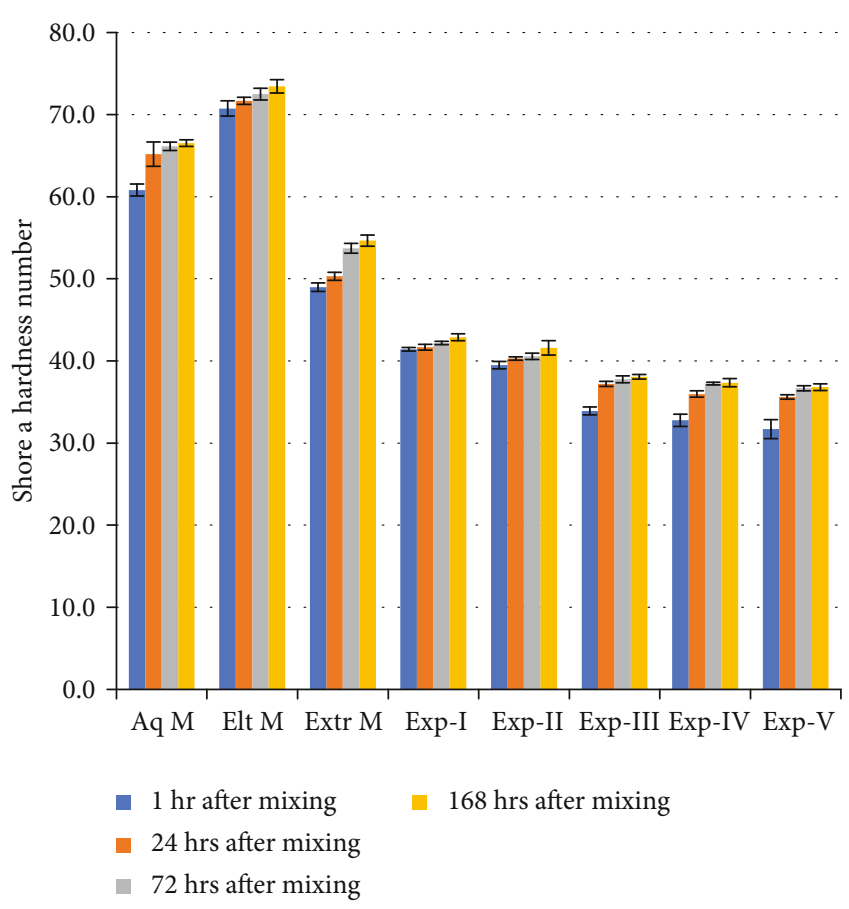

FIgURE 3: Shore A hardness of experimental and commercial VPS at four different time points ( \pm standard errors; $n=12$ ).

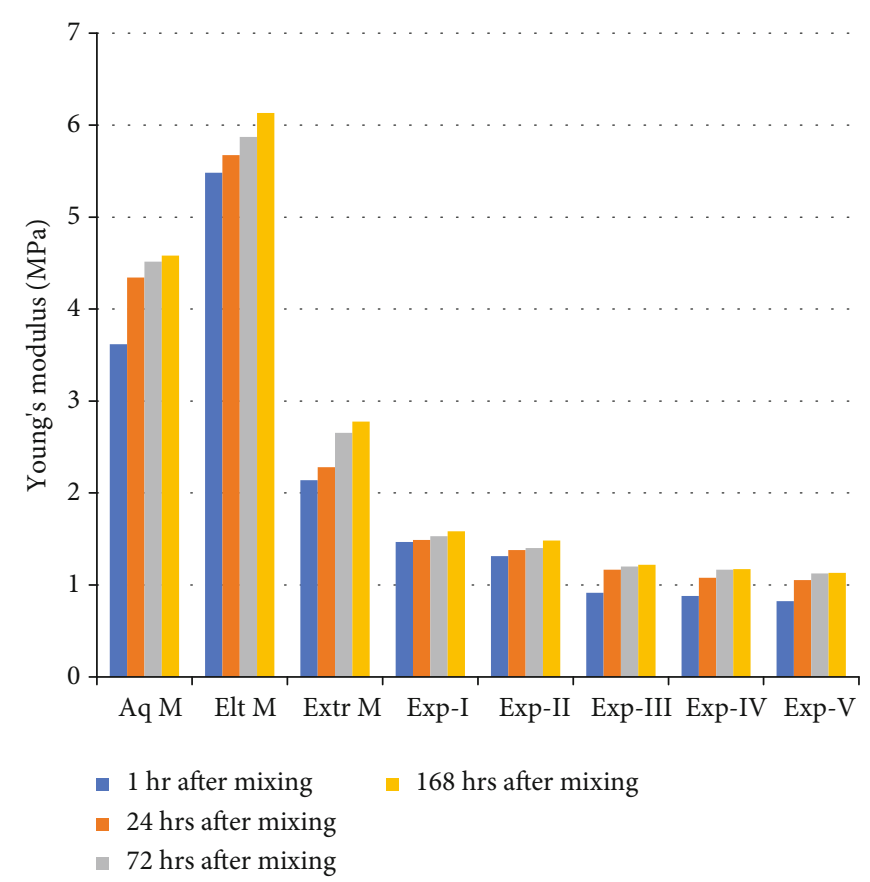

FIgURE 4: Young's modulus results of experimental and commercial VPS calculated from Shore A hardness results at four different time points.

Therefore, the hypothesis of this study is accepted. As the storage time increases so does the hardness of all the materials studied as was demonstrated by the Shore A hardness values (Figure 3 ). The cross-linking process of polymerisation continues after the material is set [21]. The increase in hardness causes an increase in the Young's (elastic) modulus $[2,3]$.

The Shore A hardness for elastomeric impression materials was in the normal range, however for experimental VPS were lower than all commercial materials at all-time points $[2,3]$. TFDMSOS in Exp-II decreased the hardness as compared to Exp-I control. The addition of surfactant (Rhodasurf CET-2) caused a further reduction in hardness. The reason for the decrease in hardness by the addition of TFDMSOS and Rhodasurf CET-2 is not clear.

Due to the molecular size of the novel cross-linking agent, the polymer chains are further apart forming a polymer matrix [22]. Increased cross-linking should improve other properties and contribute to a decrease in hardness while improving tear strength and \% elongation-at-break [23]. This data shows that the lower the hardness of the experimental VPS the higher the strain in compression values, and this inversely correlates this data.

The hardness values for all materials at all-time points were directly correlated with Young's (elastic) modulus values. After being stored at room temperature $\left(22^{\circ} \mathrm{C}\right)$ for $30 \mathrm{~min}, 60 \mathrm{~min}, 3$ hours, 24 hours, 48 hours, 1 week, and 2 weeks period time, Papadogiannis et al. [9] investigated Young's modulus of elastomeric impression materials. Their results showed that increasing storage time increased Young's moduli for all materials. The polyether material was the stiffest (10.63-12.56 MPa). The Young's moduli ranged from 1.81 to $12.99 \mathrm{MPa}$; on comparing the results of the current study with Papadogiannis et al.'s results, it was seen that Impregum F had much higher Young's moduli compared to all commercial and Exp VPS investigated in the present study (0.82 to $6.13 \mathrm{MPa})$.

ISO4823 (2007) or ADA (1977) specification 19 do not give the normal range of hardness for elastomeric impression materials, and as far as Young's modulus is concerned, there are no set criteria. There is increased difficulty in removing the impression (and tray) from the mouth $[2,3$, 24] when using stiffer impression materials with high Young's modulus.

\section{Conclusion}

(i) Commercial and experimental VPS impression materials exhibited higher Shore A hardness values with time (i.e.,1 hour after mixing, 24 hours after mixing, 72 hours after mixing, and 1 week after mixing); this was due to the continued polymerisation process after the materials had set

(ii) Shore A hardness and Young's moduli were directly proportional to each other

(iii) The hardness of the set commercial and experimental VPS materials was within the limits that set impression material can be removed from the mouth and the cast without permanent deformation

(iv) Commercial and experimental materials had enough rigidity to contain the stone during pouring 


\section{Data Availability}

The datasets used and/or analysed during the current study are available from the corresponding authors on reasonable request.

\section{Conflicts of Interest}

The authors declare no conflicts of interest.

\section{References}

[1] G. A. Helvey, "Elastomeric impression materials: factors to consider," Compendium of continuing education in dentistry (Jamesburg, NJ: 1995), vol. 32, no. 8, pp. 58-59, 2011.

[2] I. M. Meththananda, S. Parker, M. P. Patel, and M. Braden, "The relationship between Shore hardness of elastomeric dental materials and Young's modulus," Dental Materials, vol. 25, no. 8, pp. 956-959, 2009.

[3] A. Siddiqui, M. Braden, M. P. Patel, and S. Parker, "An experimental and theoretical study of the effect of sample thickness on the Shore hardness of elastomers," Dental Materials, vol. 26, no. 6, pp. 560-564, 2010.

[4] R. L. Sakaguchi and J. M. Powers, Craig's restorative dental materials-e-book, Elsevier Health Sciences, Philadelphia,PA, 2012.

[5] W. O'Brien, Dental Materials and Their Selection, Quintessence Publishing Co, Chicago, 2008.

[6] J. L. Ferracane, Materials in Dentistry: Principles and Applications, Lippincott Williams \& Wilkins, Baltimore Maryland, 2001.

[7] A. N. Gent, "On the relation between indentation hardness and Young's modulus," Rubber Chemistry and Technology, vol. 31, no. 4, pp. 896-906, 1958.

[8] R. W. Phillips, K. J. Anusavice, C. Shen, and H. Rawls, Phillips' Science of Dental Materials, Elsevier Health Sciences, St. Louis Missouri, 2013.

[9] D. Papadogiannis, R. Lakes, G. Palaghias, and Y. Papadogiannis, "Effect of storage time on the viscoelastic properties of elastomeric impression materials," Journal of Prosthodontic Research, vol. 56, no. 1, pp. 11-18, 2012.

[10] S. U. Din, N. Noor, S. Humayoun, S. Khalid, S. Parker, and M. Patel, "Tensile strength of novel experimental hydrophilic vinyl polysiloxane impression materials compared to control and commercial VPS impression materials," Journal of Islamabad Medical \& Dental College, vol. 7, no. 1, pp. 67-72, 2018.

[11] S. U. Din, S. Parker, M. Braden, and M. Patel, "The effects of cross-linking agent and surfactant on the tear strength of novel vinyl polysiloxane impression materials," Dental Materials, vol. 34, no. 12, pp. e334-e343, 2018.

[12] S. U. Din, S. Parker, M. Braden, and M. Patel, "Improved water absorption behaviour of experimental hydrophilic vinyl polysiloxane (VPS) impression materials incorporating a crosslinking agent and a novel surfactant," Dental Materials, vol. 37, no. 6, pp. 1054-1065, 2021.

[13] S. U. Din, M. Sajid, A. Saeed et al., "Dimensional changes of commercial and novel polyvinyl siloxane impression materials following sodium hypochlorite disinfection," Peer J, vol. 10, article e12812, 2022.

[14] M. Braden and J. Nicolson, Polymeric Dental Materials, Springer Verlag, Berlin, Heidelberg, 1997.
[15] International Organization for Standardization, ISO 4823, Dentistry: Elastomeric Impression Materials, International Organization for Standardization, 2000, https://cdn.standards.iteh.ai/ samples/41310/e3663121355346c29a4d04cabbfbb023/ISO4823-2000-Amd-1-2007.pdf.

[16] S. U. Din, S. Parker, M. Braden, P. Tomlins, and M. Patel, "Experimental hydrophilic vinyl polysiloxane (VPS) impression materials incorporating a novel surfactant compared with commercial VPS," Dental Materials, vol. 33, no. 8, pp. e301e309, 2017.

[17] ASTM, D 2240: standard test method for rubber property, American Society for Testing and Materials-Hardness Durometer, West Conshohoken, PA, USA, 2010.

[18] K. Grundke, S. Michel, G. Knispel, and A. Grundler, "Wettability of silicone and polyether impression materials: characterization by surface tension and contact angle measurements," Colloids and Surfaces A: Physicochemical and Engineering Aspects, vol. 317, no. 1-3, pp. 598-609, 2008.

[19] N. C. Lawson, J. O. Burgess, and M. Litaker, "Tear strength of five elastomeric impression materials at two setting times and two tearing rates," Journal of Esthetic and Restorative Dentistry, vol. 20, no. 3, pp. 186-193, 2008.

[20] N. C. Lawson, J. O. Burgess, and M. S. Litaker, "Tensile elastic recovery of elastomeric impression materials," The Journal of Prosthetic Dentistry, vol. 100, no. 1, pp. 29-33, 2008.

[21] J. McCabe and T. Carrick, "Rheological properties of elastomers during setting," Journal of Dental Research, vol. 68, no. 8, pp. 1218-1222, 1989.

[22] Z. Iqbal and M. S. Zafar, "Role of antifungal medicaments added to tissue conditioners: a systematic review," Journal of Prosthodontic Research, vol. 60, no. 4, pp. 231-239, 2016.

[23] S. Ud Din, M. Hassan, S. Khalid, M. S. Zafar, B. Ahmed, and M. Patel, "Effect of surfactant's molecular weight on the wettability of vinyl polysiloxane impression materials after immersion disinfection," Materials Express, vol. 8, no. 1, pp. 85-92, 2018.

[24] M. Braden, R. L. Clarke, J. Nicholson, and S. Parker, Polymeric Dental Materials, Springer Science \& Business Media, Berlin, Heidelberg, 2012. 